\title{
ELECTRON TRANSPORT IN THE COMA CLUSTER
}

\author{
J.G. KIRK ${ }^{1}$, P. DUFFY ${ }^{1}$, R.O. DENDY ${ }^{2}$ \\ ${ }^{1}$ Max-Planck-Institut für Kernphysik, Postfach 1039 80, \\ D-69027 Heidelberg, Germany \\ 2 UKAEA Government Division, Fusion, \\ UKAEA/Euratom Fusion Association \\ Culham, Abingdon, Oxon OX14 3DB, UK
}

\section{Introduction}

The inner regions of the Coma cluster of galaxies contain a source of diffuse synchrotron emission ('Coma C') which has a linear dimension of at least $500 \mathrm{kpc}$. There has been interest in the question of where the relativistic electrons responsible for this emission originate, and how they are transported through the intra-cluster medium (Tribble, 1993). It is widely thought that one or more of the radio galaxies in the centre of the cluster provides a likely source for particles which then diffuse out into the halo (Giovannini et al., 1993); a process which depends critically on the structure of this field in the intra-cluster medium. Recent observations of the emission from NGC4869 (Feretti et al., 1995), which occupies a central position in the Coma cluster, indicate the presence of a magnetic field which is both stronger $(B \approx 8 \mu \mathrm{G})$ and tangled on much shorter scales $\lesssim 1 \mathrm{kpc}$ than had been thought previously (Kim et al., 1990). These new results suggest not only a shorter cooling time for energetic electrons, but also a slower rate of diffusive transport. In this paper we show the constraints that the new observations place on transport theories of the relativistic electrons.

\section{Electron transport}

We consider the magnetic field to be a superposition of a regular field, $B$, and two kinds of fluctuation; microscopic and macroscopic. Microscopic fluctuations, on the scale of a particle gyroradius $\left(r_{\mathrm{g}}\right)$, result in spatial diffusion parallel and perpendicular to the magnetic field $\kappa_{\|}=\kappa_{\mathrm{B}} / \epsilon$ and $\kappa_{\perp}=$ $\epsilon \kappa_{\mathrm{B}} /(1+\epsilon)$ where $\epsilon \ll 1$ describes the amplitude of the fluctuations and 
$\kappa_{\mathrm{B}}=r_{\mathrm{g}} v / 3$ is the Bohm coefficient with $v$ the electron speed. Macroscopic fluctuations, with a scale length much larger than $r_{\mathrm{g}}$, cause the field lines to wander. In the quasilinear regime $b \equiv\left\langle\delta B^{2}\right\rangle^{1 / 2} / B \ll 1$, this can be described as a magnetic diffusion perpendicular to the average field direction with coefficient $D_{\mathrm{M}}=b^{2} \lambda_{\|} / 4$, where $\lambda_{\|}$is the correlation length along the field direction.

The effective diffusion coefficient of an electron depends on whether the mean free path for scattering on the microscopic fluctuations is longer or shorter than $\lambda_{\|}$(Duffy et al., 1995). In the former case, with $c / 2$ the average speed along a field line and $b=1$, the spatial diffusion coefficient is $D_{\text {eff }}=D_{\mathrm{M}} v=10^{31}\left(\lambda_{\|} / 1 \mathrm{kpc}\right) \mathrm{cm}^{2} \mathrm{~s}^{-1}$ independent of the magnetic field strength. Diffusive transport over a distance $R$ requires $R^{2} / D_{\text {eff }}<t_{\text {synch }}$ for $B>3 \mu \mathrm{G}$ where $t_{\text {synch }}$ is the synchrotron cooling time. Combining the above requires that $\lambda_{\|}>40 \mathrm{pc}$, in contradiction with the recent measurements, provided one identifies the correlation length with the scale of field reversals. This limitation can be avoided if the electrons are continually accelerated while they diffuse. If the microscopic fluctuations responsible for diffusion are Alfvén waves, acceleration is to be expected (Schlickeiser et al., 1987). Compensation for the synchrotron cooling requires $\kappa_{\|}=t_{\text {synch }} v_{\mathrm{A}}^{2} / 9$. In the presence of microturbulence sufficient to give the required $\kappa_{\|}$, the mean free path is shorter than the correlation length $\lambda_{\|}$, and the effective diffusion coefficient results from diffusion along field lines which themselves diffuse, a process known as 'compound diffusion' (Duffy et al., 1995): $D_{\text {eff }}=b^{4} \kappa_{\|} / 2$. The time required to propagate a distance of $500 \mathrm{kpc}$ is then $b^{-4} \times 4 \times$ $10^{11}$ years, which substantially exceeds the age of the cluster.

\section{Conclusions}

The constraints placed on quasilinear theories of electron transport in the tangled magnetic field of the Coma cluster are that with or without continuous reacceleration particles cannot be transported from the centre to the edge of the diffuse emission region. Plausible alternatives, although not without their own difficulties, include acceleration models beyond quasilinear theory or local injection and acceleration of electrons.

\section{References}

Duffy, P., Kirk, J.G., Gallant, Y.A., Dendy, R.O. $1995 A \& A, 302$, L21

Feretti, L., Dallacasa, D., Giovannini, G., Tagliani, A. $1995 A \& A, 302,680$

Giovannini, G., Feretti, L., Venturi, T., Kim, K.-T., Kronberg, P.P. 1993 ApJ, 406, 399

Kim, K.-T., Kronberg, P.P., Dewdney, P.E., Landecker, T.L. $1990 A p J, 355,29$

Schlickeiser, R., Sievers, A., Thiemann, H. $1987 A \& A, 182,21$

Tribble, P.C. 1993 MNRAS, 263, 31 